\title{
Teleportation of an arbitrary two-particle state via entanglement swapping
}

\author{
Jianxing Fang, Xianfeng Chen, Rong Zhang, and Shiqun Zhu* \\ Department of Physics, College of Sciences, \\ Suzhou University, Suzhou, Jiangsu 215006 \\ People's Republic of China
}

\begin{abstract}
A scheme of teleportation of an arbitrary two-particle state is presented when two pairs of entangled particles are used as quantum channels. After the Bell state measurements are operated by the sender, the original state with deterministic probability can be reconstructed by the receiver when a corresponding unitary transformation is followed.
\end{abstract}

Keywords: teleportation, entanglement, Bell state measurement, unitary transformation

\section{INTRODUCTION}

In recently years, much attention has been paid to quantum teleportation both theoretically and experimentally due to its important applications in quantum communication and quantum computation ${ }^{1-9}$. In the process of quantum teleportation, an unknown two-particle state, or a qubit is transmitted from a sender (Alice) to a receiver (Bob) via a quantum channel with the help of some classical information. In the original scheme proposed by Bennett et al. ${ }^{1}$, such a quantum channel is presented by a maximally entangled Bell state. Experimental realizations of teleportation were presented with the polarization photon ${ }^{2}$ and a single coherent mode of field ${ }^{3}$ in optical systems. The quantum teleportation of a two-particle entangled state by using a three-particle Greenberger-Horne-Zeilinger (GHZ) state ${ }^{4}$ as a quantum channel has been proposed ${ }^{5}$. Other schemes for teleportation of a two-particle entangled state via either a three-particle non-maximally entangled state or two non-maximally entangled particle pairs were also proposed ${ }^{6-8}$. Recently, a scheme for the teleportation of an arbitrary two-particle state was presented in Ref. ${ }^{9}$. In their scheme, two non-maximally entangled particle pairs are used as a quantum channel, and the probability of successful teleportation is determined by the smaller coefficients of the two entangled pairs.

In this letter, the teleportation of an arbitrary two-particle state via two entangled particle pairs is investigated. As the most general pure state, an arbitrary state of a two-particle system includes all kinds of two-particle states, no matter whether they are entangled. In our scheme, two maximally entangled particle pairs are used as quantum channels. After the sender (Alice) operates the Bell state measurements, the receiver (Bob) performs a corresponding unitary transformation followed by a measurement to reconstruct the original state with deterministic probability.

\section{THEORY}

An arbitrary two-particle state to be teleported can be expressed as

$$
|\psi\rangle_{12}=a|00\rangle_{12}+b|01\rangle_{12}+c|10\rangle_{12}+d|11\rangle_{12}
$$

where $|a|^{2}+|b|^{2}+|c|^{2}+|d|^{2}=1$, and $\left.\{00\rangle_{12},|01\rangle_{12},|10\rangle_{12},|11\rangle_{12}\right\}$ is the basis of a four dimensional Hilbert space. The state $|\psi\rangle_{12}$ is unknown to Alice. Without losing generality, we suppose the two entangled pairs shared by Alice and Bob are in the following states, respectively, 


$$
\begin{aligned}
& \left|\Phi^{-}\right\rangle_{34}=\frac{1}{\sqrt{2}}\left(|00\rangle_{34}-|11\rangle_{34}\right) \\
& \left|\Psi^{+}\right\rangle_{56}=\frac{1}{\sqrt{2}}\left(|00\rangle_{56}+|11\rangle_{56}\right)
\end{aligned}
$$

where $\left|\Phi^{-}\right\rangle_{34}$ and $\left|\Psi^{+}\right\rangle_{56}$ are one of the four Bell states of entangled particles 3, 4 and 5, 6 respectively. Particles 1, 2, 3 and 5 belong to the sender Alice, while particles 4 and 6 belong to the receiver Bob. Initially, the state of the system can be written as follows $|\Psi\rangle=|\psi\rangle_{12}\left|\Phi^{-}\right\rangle_{34}\left|\Psi^{+}\right\rangle_{56}$. Then, Alice operates a Bell-state measurement of particles 1 and 3 . The state of particles $2,4,5$ and 6 collapses into one of the following states,

$$
\begin{aligned}
& \left\langle\Phi^{ \pm} \mid{ }_{13} \Psi\right\rangle=\frac{1}{2 \sqrt{2}}(a|00\rangle+b|10\rangle \mp c|01\rangle \mp d|11\rangle)_{24}(|00\rangle+|11\rangle)_{56} \\
& \left\langle\Psi^{ \pm} \mid{ }_{13} \Psi\right\rangle=\frac{1}{2 \sqrt{2}}(-a|01\rangle-b|11\rangle \pm c|00\rangle \pm d|10\rangle)_{24}(|00\rangle+|11\rangle)_{56}
\end{aligned}
$$

Subsequently, Alice measures the Bell-state of particles 2 and 5 . There are probably 16 kinds of outcomes,

$$
\begin{aligned}
& \left\langle\left.\Phi^{+}\right|_{25}\left\langle\left.\Phi^{ \pm}\right|_{13} \Psi\right\rangle=\frac{1}{4}(a|01\rangle+b|00\rangle \mp c|11\rangle \mp d|10\rangle)_{46}\right. \\
& \left\langle\left.\Phi^{-}\right|_{25}\left\langle\left.\Phi^{ \pm}\right|_{13} \Psi\right\rangle=\frac{1}{4}(a|01\rangle-b|00\rangle \mp c|11\rangle \mp d|10\rangle)_{46}\right. \\
& \left\langle\left.\Psi^{+}\right|_{25}\left\langle\left.\Phi^{ \pm}\right|_{13} \Psi\right\rangle=\frac{1}{4}(a|00\rangle+b|01\rangle \mp c|10\rangle \mp d|11\rangle)_{46}\right. \\
& \left\langle\left.\Psi^{-}\right|_{25}\left\langle\left.\Phi^{ \pm}\right|_{13} \Psi\right\rangle=\frac{1}{4}(a|00\rangle-b|01\rangle \mp c|10\rangle \pm d|11\rangle)_{46}\right. \\
& \left\langle\left.\Phi^{+}\right|_{25}\left\langle\left.\Psi^{ \pm}\right|_{13} \Psi\right\rangle=\frac{1}{4}(-a|11\rangle-b|10\rangle \pm c|01\rangle \pm d|00\rangle)_{46}\right. \\
& \left\langle\left.\Phi^{-}\right|_{25}\left\langle\left.\Psi^{ \pm}\right|_{13} \Psi\right\rangle=\frac{1}{4}(-a|11\rangle+b|10\rangle \pm c|01\rangle \mp d|00\rangle)_{46}\right. \\
& \left\langle\left.\Psi^{+}\right|_{25}\left\langle\left.\Psi^{ \pm}\right|_{13} \Psi\right\rangle=\frac{1}{4}(-a|10\rangle-b|11\rangle \pm c|00\rangle \pm d|01\rangle)_{46}\right. \\
& \left\langle\left.\Psi^{-}\right|_{25}\left\langle\left.\Psi^{ \pm}\right|_{13} \Psi\right\rangle=\frac{1}{4}(-a|10\rangle+b|11\rangle \pm c|00\rangle \mp d|01\rangle)_{46}\right.
\end{aligned}
$$

where the Bell states are

$$
\begin{aligned}
& \left|\Phi^{ \pm}\right\rangle_{i j}=\frac{1}{\sqrt{2}}\left(|00\rangle_{i j} \pm|11\rangle_{i j}\right), \\
& \left|\Psi^{ \pm}\right\rangle_{i j}=\frac{1}{\sqrt{2}}\left(|01\rangle_{i j} \pm|10\rangle_{i j}\right)
\end{aligned}
$$

with $i j=13,25$.

After the measurement, Alice informs Bob of her measured results via a classical channel. Bob can reconstruct the original state only by performing a unitary transformation on his particles 4 and 6 . For instance, if Alice's first measurement result is $\left|\Psi^{-}\right\rangle_{13}$, the state of the system collapses into $\left\langle\Psi^{-} \mid{ }_{13} \Psi\right\rangle$, and the entanglement is established between particles 2 and 4 . Alice then measures the Bell-state of particles 2 and 5 . If the measurement result is $\left|\Phi^{+}\right\rangle_{25}$, the state of particles 4 and 6 collapses into the following state,

$$
\left\langle\left.\Psi^{-}\right|_{25}\left\langle\Phi^{+} \mid{ }_{13} \Psi\right\rangle=\frac{1}{4}(a|00\rangle-b|01\rangle-c|10\rangle+d|11\rangle)_{46}\right.
$$


Namely, after two measurements, the entanglement among particles 1 and 2 disappears, the new entanglement among particles 4 and 6 is set up, and the entanglement swapping occurs. Next, Alice informs Bob of her measured results by a classical channel. According to Alice's measured results, Bob operates a unitary transformation

$$
(|0\rangle\langle 1|+| 1\rangle\langle 0|)_{4} \otimes(|0\rangle\langle 0|-| 1\rangle\langle 1|)_{6}
$$

on Eq. (15). Thus entangled state in Eq. (1) can be reconstructed. Therefore, Alice successfully sends the unknown state to Bob. It is evident that bob must operate relevant unitary transformation against Alice's different measured results. Table 1 shows all different measured results by Alice and the corresponding unitary transformation by Bob.

Table 1. Measured results by Alice and relevant unitary transformation by Bob.

\begin{tabular}{|l|l|}
\hline Alice's measured results & Bob's unitary transformation \\
\hline$\left\langle\left.\Phi^{+}\right|_{25}\left\langle\left.\Phi^{ \pm}\right|_{13} \Psi\right\rangle\right.$ & $(|0\rangle\langle 0|\mp| 1\rangle\langle 1|)_{4} \otimes(|0\rangle\langle 1|+| 1\rangle\langle 0|)_{6}$ \\
\hline$\left\langle\left.\Phi^{-}\right|_{25}\left\langle\left.\Phi^{ \pm}\right|_{13} \Psi\right\rangle\right.$ & $(|0\rangle\langle 0|\mp| 1\rangle\langle 1|)_{4} \otimes(|0\rangle\langle 1|-| 1\rangle\langle 0|)_{6}$ \\
\hline$\left\langle\left.\Psi^{+}\right|_{25}\left\langle\left.\Phi^{ \pm}\right|_{13} \Psi\right\rangle\right.$ & $(|0\rangle\langle 0|\mp| 1\rangle\langle 1|)_{4} \otimes I_{6}$ \\
\hline$\left\langle\left.\Psi^{-}\right|_{25}\left\langle\left.\Phi^{ \pm}\right|_{13} \Psi\right\rangle\right.$ & $(|0\rangle\langle 0|\mp| 1\rangle\langle 1|)_{4} \otimes(|0\rangle\langle 0|-| 1\rangle\langle 1|)_{6}$ \\
\hline$\left\langle\left.\Phi^{+}\right|_{25}\left\langle\left.\Psi^{ \pm}\right|_{13} \Psi\right\rangle\right.$ & $( \pm|1\rangle\langle 0|-| 0\rangle\langle 1|)_{4} \otimes(|1\rangle\langle 0|+| 0\rangle\langle 1|)_{6}$ \\
\hline$\left\langle\left.\Phi^{-}\right|_{25}\left\langle\left.\Psi^{ \pm}\right|_{13} \Psi\right\rangle\right.$ & $(\mp|1\rangle\langle 0|+| 0\rangle\langle 1|)_{4} \otimes(|1\rangle\langle 0|-| 0\rangle\langle 1|)_{6}$ \\
\hline$\left\langle\left.\Psi^{+}\right|_{25}\left\langle\Psi^{ \pm}{ }_{13} \Psi\right\rangle\right.$ & $( \pm|1\rangle\langle 0|-| 0\rangle\langle 1|)_{4} \otimes I_{6}$ \\
\hline$\left\langle\left.\Psi^{-}\right|_{25}\left\langle\left.\Psi^{ \pm}\right|_{13} \Psi\right\rangle\right.$ & $(\mp|1\rangle\langle 0|+| 0\rangle\langle 1|)_{4} \otimes(-|0\rangle\langle 0|+| 1\rangle\langle 1|)_{6}$ \\
\hline
\end{tabular}

\section{CONCLUSION}

In conclusion, we have proposed a scheme for the teleportation of an arbitrary two-particle state via entanglement swapping. In this scheme, the quantum channel is composed of two maximally entangled particle pairs shared by sender (Alice) and receiver (Bob). Synthesizing all kinds of 16 conditions, the total probability of successful teleportation equals one. This means that complete teleportation can be realized by our method. Finally, it should be mentioned that we can select any Bell state as the quantum channel and the method in this letter can, in principle, be generalized to a $\mathrm{k}$-particle system $(\mathrm{k}>2)$.

\section{REFERENCES}

1. C.H. Bennett, G. Brassard, C. Crepeau, R. Jozsa, A. Peres and W. K. Wooters, Phys. Rev. Lett. 70, 1895 (1993).

2. D. Bouwmeester, J. W. Pan, K. Mattle, M. Eibl, H. Weinfurter and A. Zeilinger, Nature 390, 575 (1997).

3. A. Furusawa, J. L. Soensen, S. L. Brunstein, C. A. Fuchs, H. J. Kimble, E. S. Polzik, Science 282, 706 (1998).

4. M. Greenberger, M. A. Horne, A. Shimony and A. Zeilinger, J. Phys. 58, 1131 (1990).

5. V. N. Gorbachev and A. I. Trubilko, 1999 Preprint quantph/9906110.

6. W. L. Li, C. F. Li and G. C. Guo, Phys. Rev. A61, 034301 (2000).

7. H. Lu and G. C. Guo, Phys. Lett. A276, 209 (2000).

8. B. S. Shi, Y. K. Jiang and G. C. Guo, Phys. Lett. A 268, 161 (2000).

9. Y. J. Gu, Y. Z. Zheng and G. C. Guo, Chin. Phys. Lett. 18, 1543 (2001).

* szhu@suda.edu.cn; phone: (0086)-512-65226983(L); fax: (0086)-512-65111907 